\title{
ESTÁNDARES INTERNACIONALES DE EDUCACIÓN (IES) EN CONTABILIDAD Y ASEGURAMIENTO: NUEVOS RETOS DE LA PROFESIÓN CONTABLE
}

\author{
Por: Carlos Castillo Muñoz ${ }^{1}$ - Jorge Xavier Córdoba Martínez ${ }^{2}$ - José Luis Villarreal ${ }^{3}$
}

\section{RESUMEN}

La profesión contable no es ajena a los efectos de la globalización, y en sintonía con ésta, en Colombia la ley 1314 de 2009 definió la inserción del conglomerado empresarial al modelo contable internacional, más conocido como NIC-NIIF, por ello en la actualidad se desarrollan procesos de convergencia. Sin embargo, es poco lo que se ha avanzado en la determinación y acuerdos sobre la educación en contabilidad internacional y aseguramiento de futuros Contadores Públicos, y sobre la actualización de aquéllos en ejercicio.

De lo anterior, se desprende la necesidad de reflexionar sobre los aspectos vinculados a la educación superior y dar respuesta a la pregunta ¿Qué aspectos se deben tener en cuenta para la educación y formación frente al proceso de convergencia hacia estándares internacionales de información financiera y aseguramiento? Agregado a lo anterior, el propósito central es revisar las directrices de la Federación Internacional de Contadores - IFAC (Por su sigla en inglés) para la educación de los contadores profesionales.

La metodología utilizada es descriptiva, acompañada de una revisión bibliográfica reflexiva en torno a la globalización y los estándares internacionales de educación emitidos por la IFAC.

1. Contador Público, Universidad Mariana, Pasto. Magister en Gestión Empresarial, Universidad Libre, seccional Cali-Colombia. Docente-Investigador. Universidad Mariana. Pasto. Grupo de Investigación Identidad Contable. castillocarlosarbey@yahoo.com

2. Contador Público y Especialista en Pedagogía e Investigación en la Educación Superior. Universidad Mariana, Pasto-Colombia. Docente-Investigador. Universidad Mariana. Pasto. Grupo de Investigación Identidad Contable. xaviercordoba@yahoo.es

3. Contador Público, Universidad Mariana, Pasto. Magister en Gestión Empresarial, Universidad Libre, seccional Cali-Colombia. Docente-Investigador. Universidad Mariana. Pasto. Grupo de Investigación Identidad Contable. Joseluisvillarreal.di@gmail.com 
El artículo deja entrever los propósitos de tiempo atrás sobre la formación de contadores, fundamentados en el modelo global y los principales elementos de discusión al interior de las Instituciones de Educación Superior para planificar a futuro la oferta académica a nivel de pregrado y posgrado.

Palabras clave: Educación contable, estándares de educación, competencias, desarrollo profesional.

Clasificación JEL: 123.

\title{
INTERNATIONAL EDUCATION STANDARDS (IES) IN ACCOUNTING AND ASSURANCE: NEW CHALLENGES OF THE ACCOUNTING PROFESSION
}

\author{
By: Carlos Castillo Muñoz-Jorge Xavier Córdoba Martínez - José Luis Villarreal
}

\begin{abstract}
The accounting profession is no stranger to the effects of globalization, and in line with this, in Colombia the 1314/2009 law, defined the insertion of the international business conglomerate accounting model, known as IAS-IFRS, therefore at present develop convergence processes. However it is little the forward in education at international accounting and assurance of future Public Accountants, and the updating of those that are working in.
\end{abstract}

From this comes out, the need to reflect on the issues related to higher education, and to answer the following question ¿What aspects should be taken into account in the education and training of the process of convergence toward international financial reporting standards and assurance? Added to this, the main purpose is to review the International Federation of Accountants - IFAC guidelines for the education of professional accountants.

The methodology used is descriptive, accompanied by a complete bibliographic and reflexive review of the literature about globalization and international education standards issued by the IFAC.

The paper suggests the purposes of a previous time about the training of accountants, based on the global model and the main elements of discussion within the Institutions of Higher Education to plan ahead the academic offer at the undergraduate and graduate level.

Key words: Accounting education, standards of education, skills, professional development.

Classification JEL: 123. 


\section{Introducción}

Es evidente que hoy el mundo está inmerso en una serie de cambios, como resultado de un proceso económico, tecnológico, social y cultural a gran escala, consistente en la creciente comunicación e interdependencia entre los distintos países del mundo a raíz de la apertura de mercados y sociedades, que se da a través de una serie de transformaciones, económicas y políticas que les dan un carácter homogéneo; el fenómeno es conocido mundialmente como globalización; es un modelo promovido principalmente por las sociedades que viven según el sistema de democracia liberal, con el apoyo de los organismos multilaterales, tales como: el Fondo Monetario Internacional-FMI, el Banco Mundial-BM, la Organización Mundial del Comercio-OMC, el Banco Interamericano de Desarrollo-BID, la Organización para la Cooperación y Desarrollo Económico -OCDE-, la Organización de Naciones Unidas -ONU-, Conferencia de las Naciones Unidas para el Comercio y el Desarrollo -UNCTAD-, Grupo de los 20 países industrializados -G20-, entre otros, que son en los que señalan a los Estados y gobiernos de turno los ajustes en sus políticas económicas, financieras y en consecuencia sociales, que permitan incorporar las directrices emanadas desde estas instituciones.

De lo anterior, se desprende la necesidad de reflexionar sobre los elementos vinculados a la educación superior, y dar respuesta a la pregunta ¿Qué aspectos se deben tener en cuenta para la educación y formación frente al proceso de convergencia hacia estándares internacionales de información financiera y aseguramiento? Agregado a lo anterior, el propósito central es revisar las directrices de la Federación Internacional de Contadores -IFAC-, para la educación de los contadores profesionales.

El artículo está estructurado por tres apartados, el primero hace referencia a la globalización y su incidencia en la educación contable; el segundo, describe algunos elementos de la formación y educación contable; finalmente se discuten los temas propuestos.

\section{Metodología}

El diseño metodológico del artículo adopta el enfoque descriptivo formulado a partir de una revisión documental de los estándares de educación emitidos por la Federación Internacional de Contadores -IFAC-, a través del Consejo de Estándares Internacionales de educación en Contaduría -IAESB-, quien emite los Estándares Internacionales de Formación -IES-, para orientar a los programas de formación en Contaduría a nivel mundial. Para ello se analizaron y revisaron fuentes secundarias que hacen referencia al proceso de convergencia contable y su incidencia en la educación de los futuros profesionales de contaduría pública. A partir de allí se propone elementos de reflexión como aporte a la comprensión de la política estandarizadora y las posibles consecuencias sobre el modelo de educación contable colombiano. 


\section{Marco de trabajo}

\subsection{Antecedentes en educación contable}

Existe consenso en cuanto a que la educación es uno de los factores determinantes para el desarrollo, especialmente de países como Colombia, por ello pensar en el tema de educación contable, es una obligación permanente de los profesionales del área vinculados a las instituciones de educación superior; la comunidad contable, requiere reflexionar sobre las mejores prácticas para la enseñanza y aprendizaje de la contaduría pública como profesión liberal.

Para generar y desarrollar nuevas competencias en los profesionales que deben interactuar en este complejo y cambiante siglo XXI, se tendrá en cuenta las nuevas competencias como ha sido objeto de análisis por parte de distintos profesionales, entre ellos (Quijano 2002, Gracia 2002, Machado 2005, Gómez 2007, Sánchez, 2011) quienes han realizado una mirada reflexiva y critica frente a los procesos educativos, pedagógicos, curriculares y de investigación para el desarrollo de competencias.

De manera particular, la actual sociedad del conocimiento "en tanto referencia obligada en la actualidad, no sólo en el campo científico - tecnológico y de la educación, enfrenta lecturas contrapuestas frente al proceso de globalización" (Quijano, 2002: 46), situación que se constituye en un referente desde la educación contable para avanzar en la reflexión educativa con el fin de formar al contador para ser competente en ambientes de internacionalización y de una sociedad que opera en red, junto al proceso de convergencia de las nuevas dinámicas del ejercicio profesional.

Posturas más recientes, hacen referencia al reto de proponer metodologías, vinculadas a la "incorporación de metodologías activas para la enseñanza, que obedecen a los nuevos modelos pedagógicos con enfoque de competencias" (Reyes y Chaparro, 2013: 148)

\subsection{Una mirada a la globalización y su incidencia en la educación contable}

A nivel internacional se reconoce que la globalización ha producido grandes transformaciones de carácter mundial, caracterizada por la sociedad de consumo, diseñada para el auge del capital financiero, en beneficio de ciertos grupos económicos. La comprensión de este fenómeno es compleja, porque trae consigo consecuencias positivas y negativas de carácter económico, político, social y cultural.

Desde la visión unidireccional, de la política neoliberal, se promueve nuevas oportunidades del comercio, mayor acceso a los mercados y empleo de la tecnología; pero no es el caso de todos los países. Ante ellos, la globalización no escapa a las críticas de los movimientos antiglobalización que denuncian el efecto devastador que puede tener sobre las naciones en vía de desarrollo, y especialmente sobre su población vulnerable, a quienes les causa sufrimiento y detrimento en su calidad de vida (Stiglitz, 2002).

Según el premio nobel de economía Stiglitz, la consigna es clara "la globalización actual no funciona", explica que "para muchos de los pobres de la tierra no está funcionando. Para buena parte del medio ambiente no funciona. Para la 
estabilidad de la economía global no funciona. La transición del comunismo a la economía de mercado ha sido gestionada tan mal -con la excepción de China, Vietnam y unos pocos países de Europa- la pobreza ha crecido y los ingresos se han hundido" (Stiglitz, 2002: 375). De lo anterior se colige, que una causa de las grandes dificultades económicas, sociales y ambientales no es la globalización, sino el modo en que ha sido gestionada desde los organismos multilaterales (Banco Mundial -B. M-, Fondo Monetario Internacional -FMI-, Organización Mundial del Comercio -O.M.C-, -G20-, entre otros), que siempre han favorecido a los intereses de los países industrializados, particularmente de ciertas élites mundiales, en detrimento de las naciones que se encuentran en vía de desarrollo; fundamentado en un modelo económico ortodoxo, según el cual: desarrollo es vinculado al crecimiento económico y la generación de riqueza.

Conviene subrayar que la profesión contable no escapa a los efectos de la globalización, y en sintonía con ésta, se han conformado organismos supranacionales encargados de emitir diferentes tipos de estándares internacionales que todos los países del mundo deberán acoger, sin discusión alguna, esto en "apariencia" les permitirá a los países estar sincronizados con la globalización económico - financiera y ser partícipes del mercado mundial.

En el año 2010, el -G20- recalcó la importancia de establecer un único grupo de estándares globales de contabilidad mejorados y de alta calidad. Este llamado surge por las diferencias que se presentan en los sistemas de contabilidad de todo el mundo, lo que puede contribuir al surgimiento de una nueva crisis mundial, de allí se desprende la necesidad de converger en un lenguaje contable global, para disminuir las crisis financieras y minimizar su impacto en la economía mundial. Un factor que permite evitar estas crisis es el establecimiento de una nueva arquitectura financiera internacional, que promueve el -G20-, la cual se entiende como un conjunto de medidas que buscan reducir la inestabilidad financiera internacional, y también gestionar mejoras que favorezcan el crecimiento económico mundial.

Con el propósito de materializar las medidas propuestas en la nueva arquitectura financiera internacional, en el año 2009 se creó el Foro para la Sostenibilidad Financiera -FSF-, una posterior reforma hecha por el mismo lo transformó hacia una Junta de Estabilidad Financiera -FSB-, la cual promueve 12 estándares clave para configurar sistemas financieros sólidos y estables, que se agrupan en tres categorías:

a. Trasparencia de la política macroeconómica y de los datos;

b. Infraestructura institucional y de mercado; y

c. Regulación y supervisión financiera.

En contabilidad se reconoce, el Consejo de Estándares Internacionales de Contabilidad -IASB-, (por sus siglas en inglés), emisor de las Estándares Internacionales de Información Financiera -NIIF- o -IFRS- (por su sigla en inglés), Estándares Internacionales de Información Financiera para Pequeñas y Medianas Empresas NIIF para PYMES -IFRS for SMEs-, (por su sigla en inglés), lenguaje extensible de informes de negocios -XBRL- (por su sigla en inglés) estos estándares gozan de una amplia aceptación mundial. Por su parte -IFAC-, se encarga de emitir diferentes 
estándares internacionales con respecto de la profesión contable, en las siguientes áreas: Auditoria, Compromisos de Aseguramiento y Servicios Relacionados y Control de Calidad, Educación, Ética, y Contabilidad del Sector Público. Con relación al tema de Gobierno Corporativo, en el ámbito mundial se reconoce a la OCDE como emisor de los principios de buen gobierno.

En el nuevo entorno económico toma cada vez más fuerza el libre mercado, el incremento de la inversión extranjera directa, los avances tecnológicos y la volatilidad en la normatividad, basado en la economía neoliberal, como una doctrina ideológica que minimiza la intervención del Estado. En este escenario, se requiere de sistemas de información, que bajo estándares mundialmente aceptados, brinden información útil que facilite las decisiones de inversión, financiación, compra, venta, y se promueva la integración económica internacional.

En Colombia, la expedición de la Ley 1314 de 2009 definió la política de "convergencia" a estándares internacionales de contabilidad e información financiera y aseguramiento de la información, la cual facultó al Estado especialmente para intervenir la economía y así:

... expedir normas contables, de información financiera y de aseguramiento de la información, que conformen un sistema único y homogéneo de alta calidad, comprensible y de forzosa observancia, por cuya virtud los informes contables y, en particular, los estados financieros, brinden información financiera comprensible, transparente y comparable, pertinente y confiable, útil para la toma de decisiones económicas por parte del Estado, los propietarios, funcionarios y empleados de las empresas, los inversionistas actuales o potenciales y otras partes interesadas, para mejorar la productividad, la competitividad y el desarrollo armónico de la actividad empresarial de las personas naturales y jurídicas, nacionales o extranjeras.

Este aspecto motiva la reflexión sobre la forma y conveniencia de vincular estos propósitos al proceso de educación de los futuros Contadores Públicos. El cambio de modelo contable en Colombia requiere actualizar los conocimientos y mejorar las competencias de los futuros profesionales de la Contaduría Pública, quienes están llamados a liderar los proyectos de convergencia a estándares de información financiera internacional, por ello:

"La fase actual de expansión de mercados y globalización está demandando un tipo de pensamiento que se adapte al funcionamiento de sistemas económicos donde las profesiones sean cada vez más móviles y flexibles para retroalimentar el mercado de servicios profesionales" (Asfacop-RedfacontFCCCP, 2004: 145).

A lo anterior se suman las tendencias mundiales sobre educación, en tanto la Unesco determina que la educación en el siglo XXI debe fundamentarse en cuatro aprendizajes, que constituyen los pilares de formación para toda la vida: aprender a conocer, aprender a hacer, aprender a ser y aprender a vivir juntos. Desde la formación del profesional contable, el auge de la educación por competencias 
está orientado a dotar a los futuros contadores públicos, del saber hacer para el ejercicio práctico idóneo y de una actitud positiva para ser una persona capaz de vivir en sociedad y construir una mejor calidad de vida.

Una visión social e interdisciplinaria propone una comprensión desde diversos puntos de observación, por lo tanto son cuatro ejes singulares representados por: espacio, tiempo, órdenes sociales y jerarquía. La contabilidad como disciplina de conocimiento busca responder a estas dimensiones, en especial desde su vinculación con el desarrollo social, por lo tanto, "la contabilidad, considerada desde el ámbito de la información y el control, se interrelaciona necesariamente con las nuevas formas comunicadoras y controladoras del orden global” (Calvo, 2006: 210).

De ahí se infiere que la educación de contadores ahora deberá atender a las nuevas condiciones de un contexto globalizado, en el entendido que "la sociedad está convencida hoy, más que nunca, que la profesión contable es esencial para el buen funcionamiento del mercado de capitales y financiero, consecuentemente, para el buen funcionamiento de la economía” (Del Valle, 2011: 8).

\subsection{Formación y educación contable}

Se considera en principio la necesidad de distinguir entre formación y educación, como elementos básicos para entender la estandarización de las prácticas contables, y tener en cuenta las principales directrices que considera el modelo de educación de contadores promovido por la IFAC.

1.3.1 Formación versus educación. La educación y formación son dos conceptos que se deben diferenciarse a pesar de su aparente semejanza, la primera es un concepto que ha tenido una relación clara con la institución, sea está entendida como un hecho social, acciones o valores (Durkheim, 1976, citado por Quiceno, 2002: 89). La segunda es un concepto íntimamente vinculado con el sujeto, el hombre y la subjetividad (Gadamer, 1993, citado por Quiceno, 2002: 89). De lo anterior se deduce que en la educación, la persona se preocupa por obtener un título donde lo acredite para conectarse con el mundo del trabajo, bajo esa premisa asiste de manera temporal a las instituciones de educación de diversos niveles (técnico, tecnológico, profesional y posgradual) con el propósito de instruirse de forma acelerada en una práctica, ya sea técnica o profesional. En cambio, en la formación el principal interés de la persona es por su vida misma, donde realiza actividades que lo lleven a conocerse a sí mismo, la transformación de su ser, de forma

...autónoma, creativa, critica, individual y colectiva y por permitir que cada quien se dé forma, es decir, se autoconstruya como individuo para reflexionar, entre otras cosas, sobre la sujeción cultural de la que ha hecho parte en su proceso de educación (Giraldo, 2008: 11).

Una verdadera educación contable requiere los procesos de formalización que acrediten al nuevo profesional y que principalmente este acompañado de la formación en valores, análisis democrático en la sociedad que interactúa y desarrollo de un pensamiento crítico para asumir los retos de la sociedad actual, "es menester configurar nuevos escenarios y formas de concebir una educación y formación 
contable más humana y acordes con las paradojas e incertidumbres del mundo actual, que esté de parte de la libertad, la verdad, la vida, y la dignidad humana" (Freire, 2007: 27).

1.3.2 Estandarización de los procesos de educación contable. La política del Banco Mundial al inicio de la década de los 90, consistió en la adopción y aplicación de estándares internacionales, que permitieran mejorar la arquitectura financiera de los negocios, la transparencia financiera en el sector privado, el clima y niveles de inversión, aspecto que contribuiría al desarrollo de los mercados financiero y de capitales.

De esta manera, se cumple con algunos de los principios de la teoría internacional en lo relacionado con la capacidad que tienen los países desarrollados de manejar y decidir no solo las condiciones económicas y políticas de los estados en vía de desarrollo, sino la forma como deben pensar sus miembros; en ese sentido los contadores favorecerían los intereses de los grandes capitales, aunque los estándares internacionales no deben imponerse legalmente frente a las leyes y reglamentos locales, influenciarán a los organismos reguladores nacionales, a esto internacionalmente se lo conoce como endorsement, refiriéndose tanto a la adopción como a la armonización, para significar la incorporación y exigencia de los estándares internacionales (o algunos de sus criterios) al ordenamiento legal de una jurisdicción (Gómez, 2013), esto de alguna manera se convierte en un mandato que se debe cumplir.

La estandarización de la educación contable dentro del marco de la globalización busca acomodarse a esta nueva realidad, la cual exige profundas transformaciones y cambios. Sin embargo la educación fue vinculada al servicio de los grandes capitales, las fuerzas productivas y financieras, para que desde las diferentes profesiones y en particular desde la contaduría participen con su desempeño laboral en la materialización de las políticas neoliberales; "este fenómeno ha terminado por imponerse como un hecho evidente y completamente inevitable, tanto en la vida académica como en la vida cotidiana" (Quijano, 2002: 88).

A esto se añade que los organismos supranacionales y también nacionales, buscan homogenizar los procesos educativos en favor del capital financiero mundial, recalcando la visión técnico- instrumental para el profesional contable en donde el paradigma de la utilidad para la toma de decisiones se vincula al crecimiento de capital financiero y productivo en detrimento de los intereses de carácter social. En síntesis: "la contabilidad como saber estratégico siempre ha estado al servicio del capital y del poder económico; y ahora se la quiere poner al servicio del gran capital transnacional". Por ende ha ocultado los procesos inequitativos de la distribución y circulación de la riqueza (Álvarez, 2002: 179).

De otra parte se debe reconocer que a nivel mundial existen varios organismos supranacionales emisores de "directrices" internacionales para la educación de contadores profesionales; organismos como IFAC (International Federation of Accountants), ISAR (Intergovernmental Working Group Standards of Accounting), UNCTAD (United Nations Conference on Trade and Development), IMA (Institute of Management Accountants), AAA (American Accounting Association), AICPA (American Institute of Certified Public Accountants), han elaborado estudios, que al 
decir de estos organismos, proponen mejorar la educación contable bajo la premisa de un cambio para mantener su importancia y posición como profesión, así como también agregar valor a los negocios.

Por ello no debe extrañar que haya sido la IFAC quien desarrolló una propuesta de educación para Contadores Profesionales, la cual cuenta con una amplia aceptación mundial. Atendiendo a lo expresado en la misión de la IFAC: "servir al interés público, fortalecer la profesión contable en todo el mundo y contribuir al desarrollo de economías internacionales, al establecer normas profesionales de alta calidad, fomentar su cumplimiento, favoreciendo su convergencia internacional, y manifestándose sobre aquellos temas de interés público para los que la experiencia de la profesión sea más relevante" (IFAC, 2008: 2).

En el año 2003 éste organismo conformó el Consejo de Estándares Internacionales de Educación en Contaduría -IAESB-, que emite los Estándares Internacionales de Formación -IES-, para orientar a los programas de formación en Contaduría, mediante el suministro de los elementos esenciales o contenido de los programas de educación y desarrollo que permiten el reconocimiento, aceptación y aplicación internacional, según el IAESB, encargado de establecer las normas sobre la precalificación de formación, capacitación y desarrollo profesional continuo, todos los miembros ayudan a fortalecer la profesión contable, mediante las mejores prácticas en educación y desarrollo de contadores profesionales (contadores públicos en Colombia), (IFAC, 2008).

Cabe considerar que el IAESB, estableció como misión "servir al interés público a través del fortalecimiento de la profesión contable alrededor del mundo por medio del desarrollo y mejoramiento de la formación” (IFAC, 2008: 4). Para cumplir con esta misión y específicamente con su objetivo, el Consejo estableció los siguientes lineamientos estratégicos:

- El desarrollo de una serie de IES y otros pronunciamientos que reflejen la buena práctica en formación y desarrollo de contadores profesionales a nivel de pre y post-calificación;

- El desarrollo de estándares de referencia en formación a efectos de las actividades de cumplimiento de IFAC; $y$

- El fomento del debate internacional acerca de temas emergentes relacionados con formación y el desarrollo de los contadores profesionales. (IFAC, 2008: 4).

Cada uno de los pronunciamientos tiene como objetivo cumplir con una de las siguientes funciones:

- Prescribir las "mejores prácticas" (es decir, establecer las normas que los organismos miembros deben cumplir y por las cuales serán evaluados);

- Proveer de guías, interpretaciones, discusión, ilustración y ejemplos de cómo aplicar las normas, o ayudar de cualquier otra manera a los organismos miembros a alcanzar la "buena práctica"; y 
- Discutir, promover o facilitar el debate acerca de cuestiones de formación; revelar situaciones reales; presentar resultados de investigaciones o encuestas (es decir, describir situaciones); y promover la conciencia de tales cuestiones (IFAC, 2008: 4).

La estructura general de los estándares internacionales de educación emitidos por el IAESB, y publicadas en su texto oficial en el idioma inglés en el mes de agosto de 2008 son:

- Marco Conceptual de los Pronunciamientos Internacionales de Formación del IAESB.

- IES 1, Requisitos para el ingreso a un programa de formación profesional para contadores profesionales.

- IES 2, Contenido de los programas profesionales de formación en contaduría.

- IES 3, Habilidades profesionales y formación general.

- IES 4, Valores, ética y actitud profesionales.

- IES 5, Requisitos de experiencia práctica.

- IES 6, Evaluación de las capacidades y competencia profesional.

- IES 7, Desarrollo profesional continuo: un programa de aprendizaje permanente y desarrollo continuo de la competencia profesional y

- IES 8, Competencia requerida para auditores profesionales.

La temática sugerida en cada norma es amplia, pues busca regular distintos temas (o variables) de la educación profesional de los contadores. De los anteriores documentos (estándares) se resalta la importancia del marco conceptual, en él se tratan de forma general las distintas visiones y perspectivas, busca servir como base para la elaboración, desarrollo y entendimiento de los estándares internacionales de educación, y es a partir de allí donde, los programas de contaduría en conjunto, propondrán el horizonte para la profesión en Colombia.

1.3.3 Desarrollo profesional continuo. Según el marco conceptual de los IES, el desarrollo profesional continuo (DPC), y específicamente de la IES 7, se entiende como el conjunto de actividades de aprendizaje que permiten a los contadores profesionales desarrollar y mantener las capacidades para el desempeño competente en entornos profesionales; en otros términos, el DPC tiene como meta cultivar y mantener la competencia profesional después de la calificación profesional. Esto implica el desarrollo de las capacidades mediante programas de formación, formales y verificables o a través de una actividad de aprendizaje no formalizada (IFAC, 2008: 15).

Por lo tanto este IES incorpora los conceptos de desarrollo profesional continuo, como actividades de aprendizaje y resultados, que se pueden evaluar, verificar y resulten relevantes, para que los "contadores profesionales" se desarrollen competentemente en su trabajo, lo anterior, permite cumplir con el objetivo de la profesión de proporcionar servicios de alta calidad a clientes, empleadores y otros interesados. 
Además, el código de ética de IFAC para contadores profesionales establece que un contador profesional tiene el deber de mantener actualizado el conocimiento y las habilidades profesionales al nivel que sea necesario para la prestación de un servicio profesional competente.

Cabe recalcar que el DPC es parte del proceso de formación conducente a la calificación profesional, por lo tanto, el conocimiento y las habilidades profesionales, adquiridas en el momento de la calificación, siguen desarrollándose y se adaptan a las actividades y responsabilidades profesionales de la persona.

De ahí se infiere que la responsabilidad de desarrollar y mantener la competencia recae en primer lugar en cada profesional, para cumplir con ese mandato el contador profesional debe completar periódicamente un número mínimo de horas de aprendizaje, que les permitan continuar desarrollando las competencias, por ejemplo, realizar estudios formales relacionados con responsabilidades profesionales, entre otros; claro está que esos conocimientos y habilidades posteriormente se verifican mediante pruebas de evaluación para obtener la certificación.

En nuestro país se establece un sistema voluntario de educación continuada, y la responsabilidad compete exclusivamente al contador público. El código de ética profesional incluido en la ley 43 de 1990, reglamentaria de la profesión, establece en su artículo 37.7 la competencia y actualización profesional:

El Contador Público sólo deberá contratar trabajos para los cuales él o sus asociados o colaboradores cuenten con las capacidades e idoneidad necesaria para que los servicios comprometidos se realicen en forma eficaz y satisfactoria. Igualmente, el Contador Público, mientras se mantenga en ejercicio activo, deberá considerarse permanentemente obligado a actualizar los conocimientos necesarios para su actuación profesionalmente y especialmente aquellos requeridos por el bien común y los imperativos del progreso social y económico.

Desde esta perspectiva, la educación continuada es ofrecida por parte de las universidades, por medio de sus facultades de posgrados o departamentos especializados, al igual que las organizaciones profesionales de contadores, mediante sus comités de desarrollo profesional. Para cumplir lo anterior, la ley 30 de 1992, y la ley 1064 de 2006 (educación para el trabajo y el desarrollo humano), establecen diferentes niveles de educación: Diplomado, Especialización, Maestría y Doctorado. Cada una de ellas con elementos mínimos como: propósito, duración, requisitos académicos, título, énfasis investigativo o profundización.

Aquí se resalta la importancia de la educación contable como medio de recreación, contextualización y aplicación de conocimientos, en un sistema empresarial y social particular, el cual le reclama a los profesionales soluciones a los problemas que presentan en su medio, esto se puede lograr con la formación continua, que demuestren las suficientes competencias que permiten mejorar la calidad de la información financiera y a su vez, la toma decisiones del conglomerado empresarial. 


\section{Elementos de discusión}

En este documento se precisa que los aspectos a tener en cuenta para la educación y formación frente al proceso de convergencia hacia estándares internacionales de información financiera y aseguramiento, no pueden limitarse a los IES emitidos por la IFAC, los cuales como se anotó, están dirigidos principalmente a instruir a los futuros contadores en la visión técnico-instrumental al servicio del capital.

Algunos elementos que buscan ubicar la discusión en torno a una verdadera educación en la profesión contable son: estándares de educación (IES), educación por competencias y currículo

\subsection{Sobre los estándares de educación contable propuestos por la IFAC}

La propuesta de IFAC en los últimos años se ha convertido en un referente para los diferentes países que conforman el contexto de la convergencia, y hoy promueven sus estándares como las "mejores prácticas" de formación de contadores profesionales. También se identifica una posición de comodidad por parte de la comunidad contable de los países involucrados, especialmente los emergentes o en vía de desarrollo, quienes pueden llegar a considerar que los manuales contienen las soluciones a los vastos problemas que atacan a sus naciones, "todo está escrito, únicamente resta adoptar y aplicar".

En los IES se evidencia claramente la intensión de IFAC con su política estandarizadora de la educación contable internacional referida a prácticas de educación, y las consecuencias de estas prácticas en el saber contable. Escasamente promueven el desarrollo profesional de la Contaduría Pública menos el disciplinar, aunque si reproducen la visión técnica, legalista y pragmática en detrimento de su carácter científico y al servicio de la sociedad. Por su parte, la contaduría es simplificada a un oficio obediente al servicio del gran capital, sin cuestionamientos y reflexiones al mismo. En síntesis, esta propuesta centra su interés en desarrollar el mundo del hacer (Trabajo) en menoscabo del mundo del saber (Conocimiento).

Lo que promueven los estándares de IFAC, "es una civilización técnica - instrumental" (Cruz, 1994: 10) de los contadores, centrada en lo práctico, utilitario y funcional, con menos valía del conocimiento, del ser humano y la sociedad. En su propuesta "dejan entrever sus propósitos economicistas, sin mayores consideraciones acerca del saber, la investigación, la docencia, la pedagogía...” (Quijano, 2004: 103). "Su mirada es profesional-pragmática" (Gómez, 2007: 35).

En ese sentido, "la lógica de competencias planteada es adiestrante en el oficio más que legítimamente formativa en la disciplina” (Gómez, 2007: 36). Además, esta propuesta busca certificar el nivel de competencias alcanzado una vez se pongan en práctica los conocimientos adquiridos según los estándares, pues es válido afirmar que no se puede estandarizar las otras competencias mencionadas, y menos certificar competencias del mismo ser y en contextos diferentes. 
En cuanto a los contenidos propuestos por IFAC en su IES 2, está cargado de componentes técnicos, tecnológicos y financieros con énfasis en los negocios y sus necesidades económico-financieras; tiene una fuerte dosis en la capacitación en contabilidad financiera, de gestión, finanzas e impuestos. Pero no se observa componentes que permitan explicar y comprender fenómenos de lo social, ambiental, cultural, ó el mismo conocimiento contable, esto se traduce en la ausencia de campos como contabilidad social, teoría, epistemología e investigación contable.

\subsection{Educación por competencias}

Colombia, como participante de los procesos de globalización económica pasa por momentos trascendentales para modernizar y mejorar su sistema productivo y educativo, así responder de mejor manera a la dinámica de un mercado abierto, en donde los escenarios son cambiantes y las condiciones volátiles; en este contexto la profesión contable debe revisar los procesos de formación de las actuales y nuevas generaciones en armonía con los requerimientos profesionales y la educación basada en competencias.

Sin embargo, respecto al tema de competencias se presentan distintas posturas que desafían la academia para repensar los procesos formativos, "las iniciativas que vienen estructuradas en las propuestas internacionales no son necesariamente apropiadas para permitir el desarrollo de la profesión contable en armonía con el contexto empresarial y económico" (Quiroz, 2004: 150), estas condiciones llevan a pensar en que no siempre lo extranjero es lo mejor, más aún cuando fue construido para escenarios y condiciones económica y culturalmente diferentes.

La educación por competencias, no puede limitarse a la formación técnica, es necesario reconocer lo humano, aspectos sociales y comprensión del contexto, este hecho permite el desarrollo regional en diálogo con el mundo, que le permita al Contador Público contar con los elementos de juicio necesarios para asumir la realidad y fomentar el cambio y el desarrollo del capital endógeno. Es decir centrarse en las acciones locales (necesidades de educación) sin apartarse del fenómeno mundial, concepto acuñado como "glocalización" por De Sousa-Santos en el 2003.

Las educación no puede limitarse al hacer, con el fin de trasmitir conocimiento técnicos para ejecutar tareas, como lo propone la IFAC en los estándares de educación; por el contrario una formación en competencias debe vincular el ser y el conocer, la primera para promover la convivencia y el buen vivir, de tal forma que el profesional contable sea un agente social, la segunda fomenta la inteligencia cognitiva, se vincula con la investigación y promueve el desarrollo la disciplina contable.

De acuerdo con lo anterior, el debate continua abierto, la formación en competencias para los Contadores Públicos, buscará formar personas que comprendan su realidad, cercano al fenómeno de la globalización permitiendo una mejor toma de decisiones y bienestar de la comunidad, debe vincularse al "Saber-hacer y saber actuar entendiendo lo que se hace, comprendiendo cómo se actúa, asumiendo de 
manera responsable las implicaciones y consecuencias de las acciones realizadas y transformando los contextos a favor del bienestar humano" (Montenegro, 2003: 19).

Estos hechos se deben incorporar en el plan de estudio, el discurso que maneja el docente, el micro currículo (plan de cátedra), con el fin de fomentar en el futuro profesional un pensamiento reflexivo, crítico y propositivo.

Retomando las ideas sobre educación, el enfoque propuesto por la IFAC responde al modelo neoliberal de empresa de corte global; para nadie es un secreto que las propuestas de estándares para la empresas y por supuesto de educación profesional de los contadores tienen el propósito de favorecer los intereses de inversionistas financieros. A sabiendas de ello se identifica una posición de comodidad por parte de la comunidad contable de los diferentes países, especialmente los emergentes o (en vías de desarrollo), quienes asumen que la condición de homogeneidad de los estándares para las empresas y de educación, contienen al parecer las soluciones a los numerosos problemas que limitan el desarrollo de las naciones, los cuales se constituyen en dogmas, donde todo está escrito, y sólo resta adoptar y aplicar.

Por ello se ha señalado también que la intensión de IFAC con su política estandarizadora de educación, en lo que se refiere a prácticas de educación contable, y las consecuencias de estas prácticas para el saber contable, no promueven el desarrollo disciplinar de la contabilidad y tampoco profesional de la contaduría pública, aunque, si reproducen la visión técnica, legalista, práctica de la contabilidad y no su carácter científico al servicio de la sociedad. Por su parte, la contaduría es reducida a un oficio obediente al servicio del gran capital, sin cuestionamientos y reflexiones al mismo. En síntesis, esta propuesta centra su interés en desarrollar el mundo del hacer (trabajo) en detrimento del mundo del saber (conocimiento).

Las propuestas armonizadoras de mayor visibilidad en la educación contable (IFAC, ONU) prolongan el predominio de una visión profesionalizante e instrumental, la que ha eclipsado los requeridos entronques de la contaduría y la contabilidad con otras disciplinas de naturaleza social, situación que no ha viabilizado el esclarecimiento de esta como práctica social, capaz de trascender su enfoque tradicional resumido en técnica registral de amplia pobreza e indigencia conceptual y cultural. Prevalece una escasa apertura de la contabilidad a la ciencia, combatida con la inserción utilitarista fragmentada de algunas parcelas o porciones de ciencia, y con énfasis en la práctica comercial, en evidente acientificismo, y un rechazo a la interrogación conceptual y a la cultura general, y en síntesis, la no prioridad del fundamento sobre las aplicaciones, así como la parcelación absurda y la especialización funcional, de la cual sólo resultará un dislocación del conocimiento en múltiples saberes ignorantes (Quijano, 2004: 103).

Por lo tanto, es importante preguntarse respecto a la propuesta en concreto: ¿Cuáles son los argumentos que fundamentan y permiten expresar que se trata de las “mejores prácticas” de educación? ¿Qué contexto analizaron los emisores para formular dichas prácticas? ¿Qué interés incluyen estas prácticas? ¿Quién promueve la elaboración y aplicación de estas prácticas? Y si se trata de simplemente tomar un 
modelo y aplicarlo; será pertinente interrogar el “iPor qué no tomar como referente a la universidad europea o, incluso, a las americanas? ¿Por qué no estudiamos sus currículos e intentar evidenciar las lógicas y realidades a las que responden para asimilar los elementos útiles para la solución de nuestras problemáticas? ¿Por qué no estudiar el concepto de ser humano que se educa en tales regiones? ¿Será que todos a escala mundial son homo economicus irreflexivos y funcionales? ¿Por qué tienen acogida tan demagógica en nuestros países del tercer mundo estos cantos de sirena?" (Gómez, 2007: 36).

Esta serie de interrogantes constituyen el fundamento base para la reflexión e investigación profunda por parte de la comunidad contable en torno al modelo de educación propuesto por la IFAC.

Las instituciones de educación superior deberán ahondar en lo que De SousaSantos (2003) propone como "contra-hegemonía", donde se busca una relación directa de la internacionalización y globalización con la sociedad y no solamente con la empresa.

Los pilares de la formación que permitirán una educación integral tienen que ver con aspectos o conceptos poco explorados como por ejemplo la glocalidad, la cual consiste en identificar las particularidades que actores específicos tienen en los lugares geográficos directos en los que actúan las empresas o partes de ellas (De Sousa-Santos, 2003, citado por Rueda; Pinzón; Patiño, 2013: 650). Colombia es un país de regiones, especificidades, necesidades y sectores de producción y cultura; deberán ser considerados en la revisión de los currículos.

Por su parte, el modelo de formación institucional, puede ser uno propio, fundamentado en corrientes modernas como el constructivismo, es decir a partir de la construcción colectiva de la identidad, principios y valores, así el interés por los resultados de las acciones sociales y económicas son el producto de una visión compartida y una forma de ver el entorno.

Finalmente, el pilar ético, si bien lo señala IFAC debe considerarse como un problema nacional que involucra al individuo en relación con la familia, la empresa, el Estado y la sociedad, pues el ejercicio contable alrededor de las actividades económicas que terminan siendo reveladas en informes, tiene un trasfondo ético como causa y consecuencia.

La identificación y análisis de los casos concretos de antivalores que se presentan en el mundo empresarial de cada país y que han logrado disminuir la confianza en la profesión, permitirán establecer lecciones de vida para el ejercicio profesional. Es decir el pensamiento ético empieza en casa y debería alcanzar su punto más alto en los despachos del poder y en los rascacielos de las altas finanzas y la industria (Mattessich, 2004). Es preciso comprender que los acontecimientos financieros denominados "escándalos" son una manifestación de una decadencia social y cultural generalizada que obliga a cambiar los hábitos de la formación ética. 


\subsection{Corolario}

La globalización contable hace parte del proceso de internacionalización económica, unido a la emancipación del capital financiero vinculado a la ideología del modelo neoliberal; desde esta visión se busca formar un talento humano que favorezca estas actuaciones; sin embargo, la condición humanista y de carácter social de la profesión contable se deja a un lado, al centrarse en aspectos de tipo economicista, condiciones que al convertirse en el foco de atención son lesivas para fomentar el buen vivir y la sana convivencia.

No obstante, "bajo la mirada neoliberal, los efectos sociales, como aumento de la pobreza, exclusión, desigualdad y marginalización, son las consecuencias inevitables del progreso" (Rueda, 2010: 154), entonces la educación no puede limitarse a las prácticas de estandarización contable, administrativa y de aseguramiento (NIIF, NIA, gobierno corporativo, control interno, gestión estratégica y cuadro de mando integral), debe entenderse que existen diversas y complejas dimensiones, incluyendo asuntos políticos, tecnológicos, culturales y educativos en lo social, buscando la defensa del interés público, en donde prime el resultado por la construcción y comprensión del rol social, institucional y político de la contabilidad como disciplina de conocimiento.

Es hora de comprender que la condición de homogenizar las prácticas contables y educativas del profesional, limitada a lo tecnocrático y mercantil no promueve la construcción y desarrollo de soluciones para avanzar en la equidad social. Hoy los procesos educativos deben incrementar la problematización de la disciplina y las organizaciones dentro y fuera del aula, en cuanto la sociedad requiere personas que promuevan la defensa de lo público y la generación de alternativas de solución, que se reconoce en sociedad y resalta su identidad, trabaja con otras personas para generar cambios en su entorno, desde las dimensiones social, cultural y ambiental a fin de fomentar el desarrollo equitativo y la inclusión social.

\section{Conclusiones}

La visión ortodoxa de la economía considera que la política neoliberal genera nueva oportunidades de mercado, pero no es el caso para todos los países; sin embargo los procesos de integración internacional vigentes en Colombia atendiendo a los intereses de ciertos grupos de interés, en el año 2009 dieron como resultado la expedición de la ley 1314 con la cual se generan cambios en el modelo contable y además requiere la actualización de los conocimientos y mejoramiento de las competencias de los futuros profesionales de la Contaduría Pública, es decir exige por lado la adaptación al sistema económico y por otro la retroalimentación del mercado de los servicios profesionales.

Los estándares de educación formulados por la IFAC prolongan el predominio de una visión profesionalizante y de carácter instrumental, este modelo está cargado de componentes técnicos, tecnológicos y financieros con énfasis en los negocios y sus necesidades económico-financieras, pero no se observa componentes que 
permitan explicar y comprender fenómenos de lo social, ambiental, cultural, o el mismo conocimiento contable, esto se traduce en la ausencia de campos como contabilidad social, teoría, epistemología e investigación contable.

Los planes y programas de educación contable en los niveles de pregrado deberán ser revisados para rescatar aquello que otrora permitía enfocarlos en la naturaleza mutua de las profesiones, es decir al servicio de la sociedad, cimentada en valores y con amplio sentido de la democracia, la dignidad humana y el medio ambiente. A su vez la educación continua a cargo de las facultades de posgrado recabará en los requerimientos sociales-empresariales para los profesionales en ejercicio y el Estado promoverá la cualificación más allá de la voluntad o el propio interés de quienes ostentan la facultad de otorgar fe pública.

En últimas, el rescate del interés público en la formación del Contador, se debe enfocar por una educación capaz de generar alternativas a fin de ser partícipe de los procesos de transformación que cotidianamente mueven hacia un mundo más humano-solidario y menos centrado en lo material-económico.

\section{Referencias}

ÁLVAREZ, A. Harold (2002). Adopción o armonización de la normativa contable. . En: Revista cuadernos de administración. No. 28, diciembre de 2002.

ASFACOP-REDFACONT-FCCCP (2004). Marco conceptual de fundamentación de la prueba. Tomo I.

CALVO V., Patricia. La globalización y su relación con la contabilidad. Revista Facultad de ciencias económicas. Investigación y reflexión. Vol. 14 No. 1, junio de 2006.

CONGRESO DE LA REPÚBLICA. Ley 1314 de 2009, por la cual se regulan los principios y normas de contabilidad e información financiera y de aseguramiento de la información aceptada en Colombia. Diario Oficial, 47.409, 13 de julio de 2009. Disponible en: http://docs.colombia.justia.com/nacionales/leyes/ ley-1323-de-2009.pdf (Consultado 04-09-2014).

CONGRESO DE LA REPÚBLICA. Ley 43 de 1990, por la cual se adiciona la ley 145 de 1960, reglamentaria de profesión de contador público. Disponible en: http://www.mineducacion.gov.co/1621/articles-104547_archivo_pdf.pdf (Consultado 04-09-2014).

CONGRESO DE LA REPÚBLICA. Ley 30 de 1992, por la cual se organiza el servicio público de la Educación Superior. Disponible en: http://www.mineducacion.gov.co/1621/articles-86437_Archivo_pdf.pdf (Consultado 04-09-2014). 
CONGRESO DE LA REPÚBLICA. Ley 1064 de 2006. Por la cual se dictan normas para el apoyo y fortalecimiento de la educación para el trabajo y el desarrollo humano establecida como educación no formal en la Ley General de Educación. Disponible en: http://www.mineducacion.gov.co/1621/articles-104704_archivo_pdf.pdf (Consultado 04-09-2014).

CRUZ K., Fernando (1994). La Sombrilla Planetaria, Ensayos sobre Modernidad y Postmodernidad, en: La Cultura. Bogotá: Planeta, 1994.

DEL VALLE, Fermín (2008). La profesión contable: su rol en la sociedad. Revista internacional Legis de contabilidad y auditoría. No. 36. Bogotá. Editorial Legis. Abril-junio de 2008.

FEDERACIÓN INTERNACIONAL DE CONTADORES - IFAC (2008). Manual de los Pronunciamientos Internacionales de Formación.

FREIRE, Pablo (2007). La educación como práctica de la libertad. México. Siglo XXI Editores.

GÓMEZ V., Mauricio (2007). Las insuficiencias disciplinares de los entandares internacionales de educación -IES- para contadores profesionales. En: Revista Latinoamericana de pensamiento, teoría e investigación contable. LUMINA. No. 07. Facultad de Contaduría Pública. Universidad de Manizales. Manizales.

GÓMEZ V., Mauricio (2013). La reforma de la gestión pública en Latinoamérica:su impacto en la Transparencia y la divulgación de la información financiera. Tesis doctoral.

GIRALDO G., Gregorio (2008). Prólogo. Unidad gremial: una alternativa para la construcción de un proyecto de nación. En: XX Congreso Nacional de Estudiantes de Contaduría Pública. ASECUVA-FENECOP. Cali. 2008.

MATTESSICH, Richard (2004). Lecciones de Enron y Arthur Andersen Co. Universidad de Málaga. Madrid.

PUC (2012). Plan único de cuentas para comerciantes. Editorial Legis, Bogotá.

MONTENEGRO, Ignacio (2003). ¿Son las competencias el nuevo enfoque que la educación requiere?. En: Magisterio: Educación y Pedagogía, No. 1.

QUICENO C., Humberto (2002). Educación y formación profesional. Facultad de ciencias de la administración. Universidad del Valle. En: Revista Cuadernos de Administración. No. 28, diciembre de 2002.

QUIJANO V., Olver, et al. (2002). Del Hacer al Saber. Realidades y perspectivas de la educación contable en Colombia. Popayán Editorial Universidad del Cauca.

QUIJANO V., Olver (2004). Estandarización de la educación contable: El proyecto escolar de los gremios económicos. En: La contaduría pública en el nuevo entorno global. Rutas y desviaciones. Universidad del Cauca - Centro Colombiano de Investigaciones Contables. Editorial Universidad del Cauca. Popayán.

QUIROZ, Elkin (2004). Competencias formativas y mercado profesional contable. En: Revista Internacional Legis de Contabilidad \& Auditoria. No. 32. Legis Editores. Bogotá, Colombia (143- 196).

REYES, Nydia; CHAPARRO, Fernando (2013). Metodologías activas para la enseñanza de las Normas Internacionales de la Información Financiera en un ambiente virtual de aprendizaje. Revista Cuadernos de Contabilidad. Vol. 14 No. 36.

RUEDA, Gabriel (2010)., El papel de la contabilidad ante la actual realidad económica, social y política del país. Más allá de la convergencia de prácticas mundiales. En: Revista Cuadernos de Contabilidad. Vol. 11 No. 28. Bogotá.

RUEDA D., Gabriel, PINZÓN, Jorge; PATIÑO, Ruth (2013). Los currículos de los programas académicos de contaduría pública, tras la enseñanza de lo internacional y la globalización en la contabilidad: necesidades de ajuste más allá de las respuestas técnicas. Cuadernos de contabilidad, Volumen 14, No. 35. Julio-diciembre de 2013.

STIGLITZ, Joseph E. (2002/2008). El malestar en la globalización. Madrid: Santillana Ediciones Generales, S.L. 